\title{
Clinical Prediction Rule for Developmental Delay at 3 Years of Age among Very Low Birth Weight Infants
}

\author{
Masayo Kokubo1,2, Tomohiko Nakamura1', Sayaka Shimizu², Miho Kimachi², Shunichi Fukuhara² \\ ${ }^{1}$ Nagano Children's Hospital, Neonatology, Azumino, Japan, ${ }^{2}$ Kyoto University, Healthcare Epidemiology, Kyoto, Japan
}

\section{Background and Aims}

Very low birth weight infants (VLBWI) are at high-risk of neurodevelopmental impairment. Early developmental intervention has been recognized to prevent motor and cognitive impairment. Although we can anticipate that severe brain injuries cause sequelae, we have no useful prediction rule include enough perinatal predictors for developmental delay among VLBWI without severe brain injuries. The aims of this study are to develop and validate clinical prediction rule for developmental delay among VLBWI without severe brain injuries at the time of NICU discharge.

\section{Methods}

Design: Retrospective cohort study.

Setting: The multicenter NICU in Japan.

Participants: Among 10,195 VLBWI during 2003-2010, a prediction rule was derived identifying infants with developmental delay. Validation was internal among 3,581 VLBWI during 2011-2012. Infants with grade III or IV intraventricular hemorrhage (IVH), cystic periventricular leukomalacia, posthemorrhagic hydrocephalus or hypoxic-ischemic encephalopathy were excluded.

Main outcome: Developmental delay at 3 years of age defined by an overall developmental quotient $<70$ as measured by Kyoto Scale of Psychological Development test. Discrimination was assessed by the area under the receiver operating characteristic curves (AUC).

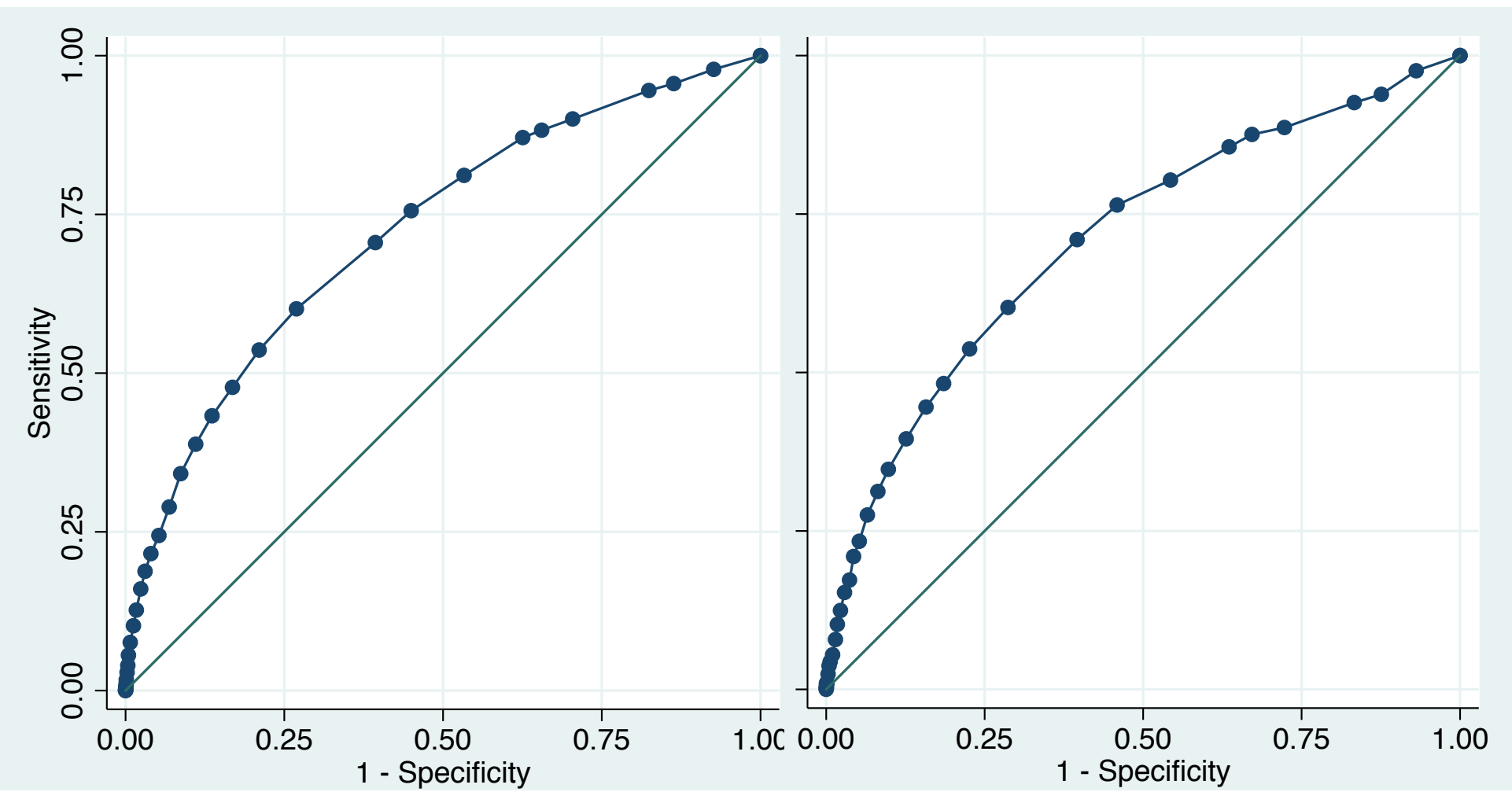

Figure. Discrimination of prediction model. Left. AUC of the model in derivation set was 0.72 . Right. AUC of the model in validation set was 0.71 .

\section{Conclusion}

Among VLBWI without neonatal severe brain injuries, clinical prediction rule for developmental delay may be useful for early developmental intervention.

\section{Results}

The prevalence of developmental delay in derivation and validation set were $13 \%$ and $15 \%$, respectively. We developed a clinical prediction rule included 10 predictors; gestational age (GA), gender, small for gestational age (SGA), severe bronchopulmonary dysplasia (BPD), inflammation consist of necrotizing enterocolitis (NEC), focal intestinal perforation (FIP) or sepsis, Apgar score at 5 min, grade I or II IVH, symptomatic patent ductus arteriosus (PDA), severe retinopathy of prematurity (ROP) and adrenal insufficiency of prematurity. AUC of the model in derivation and validation set were 0.72 and 0.71 , respectively.

Table. Score of 10 predictors for developmental delay

\begin{tabular}{|c|c|c|c|c|}
\hline Predictors & Category & Score & $\mathbf{P}$ & $95 \% \mathrm{Cl}$ \\
\hline \multirow[t]{5}{*}{ GA } & $30 w \leqq$ & 0 & - & \\
\hline & $28-29 w$ & 1 & 0.295 & -0.0910 .301 \\
\hline & $26-27 w$ & 3 & 0 & 0.2410 .656 \\
\hline & $24-25 w$ & 6 & 0 & 0.6211 .099 \\
\hline & $22-23 w$ & 9 & 0 & 1.0761 .709 \\
\hline Sex & Male & 6 & 0 & 0.7340 .995 \\
\hline SGA & + & 3 & 0 & 0.3410 .649 \\
\hline $\begin{array}{l}\text { Adrenal } \\
\text { insufficiency }\end{array}$ & + & 1 & 0.143 & -0.0510 .355 \\
\hline \multirow[t]{3}{*}{ Severe BPD } & - & 0 & - & \\
\hline & +/HOT- & 1 & 0.091 & -0.0240 .326 \\
\hline & $+/ \mathrm{HOT}+$ & 7 & 0 & 0.7751 .237 \\
\hline $\begin{array}{l}\text { ROP needed } \\
\text { treatment }\end{array}$ & + & 2 & 0.001 & 0.1250 .452 \\
\hline IVH & + & 2 & 0.007 & 0.0760 .471 \\
\hline \multirow[t]{3}{*}{$\begin{array}{l}\text { Apgar score } \\
\text { at } 5 \mathrm{~min}\end{array}$} & $7-10$ & 0 & & \\
\hline & $4-6$ & 1 & 0.012 & 0.0440 .355 \\
\hline & $0-3$ & 2 & 0.050 & $0.000 \quad 0.571$ \\
\hline $\begin{array}{l}\text { NEC/FIP } \\
\text { /Sepsis }\end{array}$ & + & 5 & 0 & 0.4970 .883 \\
\hline \multirow[t]{3}{*}{$\begin{array}{l}\text { Symptomatic } \\
\text { PDA }\end{array}$} & - & 0 & & \\
\hline & $\begin{array}{l}\text { Indomethacin+ } \\
\text { /surgery- }\end{array}$ & 1 & 0.143 & -0.0360 .251 \\
\hline & $\begin{array}{l}\text { Indomethacin+ } \\
\text { /surgery+ }\end{array}$ & 2 & 0.015 & 0.0600 .545 \\
\hline
\end{tabular}

